\title{
Differential requirement for NO during ABA-induced stomatal closure in turgid and wilted leaves
}

\author{
DIMAS M. RIBEIRO ${ }^{1,2}$, RADHIKA DESIKAN ${ }^{3}$, JO BRIGHT $^{2}$, ANA CONFRARIA $^{2,4}$, JUDITH HARRISON $^{2}$, \\ JOHN T. HANCOCK ${ }^{2}$, RAIMUNDO S. BARROS ${ }^{1}$, STEVEN J. NEILL ${ }^{2} \&$ IAN D. WILSON $^{2}$ \\ ${ }^{1}$ Departamento de Biologia Vegetal, Universidade Federal de Viçosa, Viçosa 36571-000, Minas Gerais, Brazil, ${ }^{2}$ Centre for \\ Research in Plant Science, Faculty of Health and Life Sciences, University of the West of England, Bristol BS16 1QY, UK, \\ ${ }^{3}$ Division of Biology, Faculty of Natural Sciences, Imperial College, London SW7 2AZ, UK and ${ }^{4}$ Departamento de Botânica, \\ Faculdade de Ciêncas, Universidade do Porto, 4169-007 Porto, Portugal
}

\begin{abstract}
Abscisic acid (ABA)-induced stomatal closure is mediated by a complex, guard cell signalling network involving nitric oxide (NO) as a key intermediate. However, there is a lack of information concerning the role of NO in the ABAenhanced stomatal closure seen in dehydrated plants. The data herein demonstrate that, while nitrate reductase (NR)1-mediated NO generation is required for the ABAinduced closure of stomata in turgid leaves, it is not required for ABA-enhanced stomatal closure under conditions leading to rapid dehydration. The results also show that NO signalling in the guard cells of turgid leaves requires the ABA-signalling pathway to be both capable of function and active. The alignment of this NO signalling with guard cell $\mathrm{Ca}^{2+}$-dependent/independent $\mathrm{ABA}$ signalling is discussed. The data also highlight a physiological role for NO signalling in turgid leaves and show that stomatal closure during the light-to-dark transition requires NR1mediated NO generation and signalling.
\end{abstract}

Key-words: abscisic acid; nitric oxide; stomata; transpiration; water stress.

\section{INTRODUCTION}

The reduced availability of water during periods of drought has adverse effects on the growth and productivity of crop plants. In vegetative tissues, water stress may lead to a reduction in cell turgor and signals which are transduced into the enhanced biosynthesis and redistribution of abscisic acid (ABA), resulting in a variety of ABA-mediated responses such as altered gene expression and stomatal closure (Nambara \& Marion-Poll 2005). Recent work has demonstrated that the stomatal responses of Arabidopsis plants can be manipulated by modifying guard cell signal transduction elements in order to reduce the water lost during periods of water deficit (Saez et al. 2006).

Among the components participating in ABA-activated signalling pathways which lead to stomatal closure, nitric

Correspondence: I. D. Wilson. Fax: +44 (0)11732 82799; e-mail: ian2.wilson@uwe.ac.uk oxide (NO) is an important intermediate (Garcia-Mata \& Lamattina 2002; Neill et al. 2002, 2008). ABA-induced NO synthesis in guard cells is associated with stomatal closure in a number of species (Neill, Desikan \& Hancock 2003; Desikan et al. 2004) and the importance of NO has been further substantiated by employing NO donors such as sodium nitroprusside (SNP) which cause stomatal closure and NO scavengers such as 2-phenyl-4,4,5,5tetramethylimidazoline-1-oxyl-3-oxide (PTIO) which impair ABA-induced stomatal closure (Desikan et al. 2002; Garcia-Mata \& Lamattina 2002; Neill et al. 2002; Bright et al. 2006). Such studies have demonstrated that endogenous $\mathrm{NO}$, generated in response to $\mathrm{ABA}$, is required for stomatal closure to occur.

Genetic evidence has also confirmed the role of NOgenerating enzymes in mediating stomatal closure in response to ABA. Stomata of the Arabidopsis double mutant, nial nia2, which is deficient in the nitrate reductase apoproteins, nitrate reductase (NR)1 and NR2, do not close in response to either $\mathrm{ABA}$ or the substrate, nitrite, from which NR generates NO (Desikan et al. 2002). There have also been a number of suggestions that NO may protect plants against water stress (Garcia-Mata \& Lamattina 2001; Tian \& Lei 2006). However, despite the ABA insensitivity shown by its guard cells, the nia1 nia2 double mutant does not show a wilty phenotype (Desikan et al. 2002). Thus, the exact contribution made by ABA-induced guard cell NO synthesis with regard to water-stress tolerance is unclear. A re-evaluation of the mechanism of ABA-induced stomatal closure via NO synthesis is therefore necessary to elucidate the exact role played by NO during responses to ABA and water stress in guard cells. Here, we show that while NO has a role to play in the movement of guard cells in wellhydrated tissues, it appears not to be required for the closure of stomata during periods of dehydration.

\section{MATERIALS AND METHODS}

\section{Plant material and growth conditions}

Seeds of wild-type Columbia (Col-0) and Landsberg erecta (Ler) ecotypes of Arabidopsis thaliana and mutants of each of these were germinated on Levington's F2 compost with 
sand (Avoncrop, Bristol, UK) and the plants grown in growth chambers (Sanyo Gallenkamp, Loughborough, UK) under a $16 \mathrm{~h}$ photoperiod $\left(60-100 \mu \mathrm{E} \mathrm{m}^{-2} \mathrm{~s}^{-1}\right)$ at $20^{\circ} \mathrm{C}$ with $70-80 \%$ relative humidity. Plants were used at 4 weeks postgermination. The nia1::Ds (NR1 deficient; background Ler) and nia2-5 (NR2 deficient, background Col-0) mutant seeds were obtained from Prof Nigel Crawford (University of California, San Diego, CA, USA); nia1 nia2 (NR1,2 deficient; background Col-0), abil-1 (ABA insensitive 1; background Ler) and aba1-1 ( $A B A$ deficient 1; background Ler) mutant seeds were obtained from the Nottingham Arabidopsis Stock Centre, Nottingham, UK. In all experiments using mutants, the appropriate background was used as the wild-type control.

\section{Stomatal bioassays}

Stomatal bioassays were performed using leaves as described by Desikan et al. (2006). Leaves detached from 4-week-old plants were floated in 2-(Nmorpholino)ethanesulfonic acid (MES)-KCl buffer $(5 \mathrm{~mm}$ $\mathrm{KCl}, 10 \mathrm{~mm}$ MES, $50 \mu \mathrm{M} \mathrm{CaCl}_{2}$, $\mathrm{pH}$ to 6.15 using dilute Tris base solution) with abaxial side down in Petri dishes (30 $\mathrm{mm}$ diameter) and incubated under continuous illumination $\left(60-100 \mu \mathrm{E} \mathrm{m}^{-2} \mathrm{~s}^{-1}\right)$ at $20{ }^{\circ} \mathrm{C}$, for $2.5 \mathrm{~h}$ to open the stomata. Following this, leaves were treated with various compounds supplemented in the same buffer medium for a further $2.5 \mathrm{~h}$. Inhibitor compounds, either PTIO or 2-(4carboxyphenyl)-4,4,5,5-tetramethylimidazoline-1-oxyl-3oxide (cPTIO), were added to the MES-KCl buffer medium in which leaves were floated $30 \mathrm{~min}$ prior to other treatments. The leaves were subsequently homogenized individually with approximately $250 \mathrm{~mL}$ water in a Waring blender (Christison Scientific, Gateshead, UK) for 30 s. The epidermal fragments were collected on a $100 \mu \mathrm{m}$ nylon mesh (Spectra-Mesh, BDH-Merck, Nottingham, UK), transferred onto a glass slide and were covered with a glass slip. Stomatal apertures from epidermal fragments were then measured using a calibrated light microscope coupled to an imaging system (LEICA QWIN Software, Leica, Milton Keynes, UK).

To assess the effect of the various compounds on stomatal closure under water-stress conditions, detached leaves were floated on MES-KCl buffer medium under light for $2.5 \mathrm{~h}$ to open the stomata and then the compounds were added to the buffer solution. Thereafter, a $2.5 \mathrm{~h}$ drought stress was performed by placing detached leaves with abaxial side facing upwards on open Petri dishes on a paper layer (Whatman number 1, Whatman International Ltd., Maidstone, Kent, UK) under a direct light source $(50 \mathrm{~cm}$ from a $60 \mathrm{~W}$ bulb) on the laboratory bench, for $2.5 \mathrm{~h}$. The leaves were subsequently blended and the resulting epidermal fragments were taken for immediate examination of stomatal apertures as described above.

To assess the effect of the light-to-dark transition, detached leaves were floated on MES-KCl buffer medium under light for $2.5 \mathrm{~h}$ to open the stomata, at which time, different compounds were added to the media and the leaves were placed in the dark for $2 \mathrm{~h}$ before being assayed for stomatal aperture as before. To assess the effect of different compounds on light-induced stomatal reopening during the dark-to-light transition, detached leaves were floated on MES-KCl buffer medium in the dark for $2 \mathrm{~h}$, at which time, various compounds were added to the medium and the leaves were incubated under light for a further $2 \mathrm{~h}$. After incubation, stomatal apertures were assayed as before.

\section{Confocal microscopy}

NO was visualized by confocal microscopy using 4,5diaminofluorescein diacetate [(DAF2-DA); Calbiochem, Nottingham, UK]. Leaves were homogenized individually with water in a Waring blender (Christison Scientific) for $30 \mathrm{~s}$. The epidermal fragments were collected on a $100 \mu \mathrm{m}$ nylon mesh (Spectra-Mesh, BDH-Merck) and transferred to $2 \mathrm{~mL}$ MES-KCl buffer in a Petri dish. Epidermal fragments were incubated under a direct light source for $2 \mathrm{~h}$ and were then subsequently loaded with $15 \mu \mathrm{m}$ DAF2-DA for $15 \mathrm{~min}$ and then washed with MES-KCl buffer for $20 \mathrm{~min}$. Following this, fragments were treated with ABA or nitrite supplemented in the MES-KCl buffer for a further $25 \mathrm{~min}$. Confocal laser scanning microscopy (Nikon PCM 2000; Nikon, Kingston-upon-Thames, UK) was used to visualize DAF2-DA fluorescence (excitation $488 \mathrm{~nm}$, emission $515 \mathrm{~nm})$.

For the observations of dark-induced NO-associated fluorescence, leaves were blended as before and the collected fragments were equilibrated with or without cPTIO, were loaded with DAF-2DA in the dark for $15 \mathrm{~min}$, washed with $\mathrm{MES}-\mathrm{KCl}$ buffer as before but in the dark, and were then continuously incubated in the dark until a total dark period of $2 \mathrm{~h}$ after the onset of dye loading was attained. The fluorescence was then observed as before.

Images acquired from the confocal microscope were analysed using SCION IMAGE software (Scion Corp, Frederick, MA, USA) to quantify the total fluorescence of guard cells in terms of pixel intensities. Data are presented as mean pixel intensities with standard errors. Mean emission autofluorescence background of guard cells in the absence of DAF-2DA and mean background DAF2-DA fluorescence taken from clear parts of the glass slides have been removed from the data.

\section{Measurement of water loss}

Leaves were floated in MES-KCl buffer for $2.5 \mathrm{~h}$ under illumination (60-100 $\mu \mathrm{E} \mathrm{m}^{-2} \mathrm{~s}^{-1}$ ) in a growth chamber. Following this, leaves were treated with ABA, SNP or nitrite and were incubated in the same buffer for a further $2.5 \mathrm{~h}$. Water loss was measured by weighing with time detached leaves placed abaxial side up in open Petri dishes $(30 \mathrm{~mm}$ diameter) on a layer of filter paper under a direct light source. The weight of each set of leaves was determined every $20 \mathrm{~min}$ over a period of $3 \mathrm{~h}$. Water loss was expressed as the percentage of the initial weight. 


\section{Drought stress in whole plants and relative water content (RWC) measurement}

At 4 weeks post-germination, the aerial parts of wellwatered Arabidopsis plants were subsequently submerged for $5 \mathrm{~s}$ each day for a further $3 \mathrm{~d}$ either in MES-KCl buffer or in solutions of either ABA $(50 \mu \mathrm{M})$ or SNP $(50 \mu \mathrm{M})$ prepared in MES-KCl buffer. After this 3 day period of pre-treatment, drought was induced by withholding the watering of plants maintained in the growth chamber (16 h photoperiod, $60-100 \mu \mathrm{E} \mathrm{m}^{-2} \mathrm{~s}^{-1}, 20{ }^{\circ} \mathrm{C}, 70-80 \%$ relative humidity). To minimize experimental variation, plants were grown individually in $0.17 \mathrm{~L}$ plastic pots. Non-plant evaporation from the pots was minimized by covering the exposed soil surface with plastic film (Parafilm 'M', Chicago, IL, USA) during the water-stress treatments. At each time point, 10 leaves from four different plants were sampled after each period of drought and their fresh weights (FW) immediately recorded. Subsequently, the detached leaves were rehydrated in distilled water for $3 \mathrm{~h}$ in the growth chamber and their recovered turgid weight (TW) was recorded. After drying for $24 \mathrm{~h}$ at $80^{\circ} \mathrm{C}$, the dry weight (DW) of the leaves was also recorded. The RWC of the leaves were then estimated according to the formula: $\mathrm{RWC}(\%)=[(\mathrm{FW}-\mathrm{DW}) /(\mathrm{TW}-\mathrm{DW})] \times 100$.

\section{Statistical analysis}

Data from stomatal aperture assays and RWC and water loss data were subjected to analysis of variance where $P>0.05$ was considered as being not significant. Differences in the between treatment means of stomatal apertures and water losses were examined by the $t$-test $(P \leq 0.05)$. In the

Figure 1. (a) Nitric oxide (NO) is only required for stomatal closure during turgid but not dehydrated conditions. Wild-type Arabidopsis [Columbia (Col-0)] leaves were floated in 2-(N-morpholino)ethanesulfonic acid (MES)-KCl buffer under light to induce stomatal opening and then incubated for $2.5 \mathrm{~h}$ in buffer alone (control), $50 \mu \mathrm{M}$ abscisic acid (ABA), $50 \mu \mathrm{M}$ sodium nitroprusside (SNP), $200 \mu \mathrm{M}$ 2-phenyl-4,4,5,5-

tetramethylimidazoline-1-oxyl-3-oxide (PTIO) or ABA + PTIO. Stomatal apertures were measured before dehydration (white bars) and 30 min after submitting the leaves to dehydration (black bars) under a direct light source. The data are representative of four independent experiments with means of 100 stomata \pm standard errors. (b) NO is not required for ABA-induced water-stress tolerance. Wild-type Arabidopsis (Col-0) leaves were floated in MES-KCl buffer under light to induce stomatal opening and then incubated for $2.5 \mathrm{~h}$ in buffer alone (Control), $50 \mu \mathrm{m}$ ABA, $50 \mu \mathrm{m}$ SNP, $200 \mu \mathrm{m}$ PTIO,

$\mathrm{ABA}+\mathrm{SNP}, \mathrm{ABA}+\mathrm{PTIO}, \mathrm{SNP}+\mathrm{PTIO}$ or ABA + SNP + PTIO.

Wilting was induced by placing detached leaves with abaxial side up in open Petri dishes under a direct light source. Relative water content (RWC) values were determined after $2 \mathrm{~h}$ of applying the drought condition. Data are means of two independent experiments with four replicates (Petri dishes with four leaves) for each treatment \pm standard errors. Bars labelled with the same letter are not statistically different by Tukey test $(P<0.05)$. case of the RWC assays, mean comparisons were performed using the Tukey test. All mean comparisons were performed with the statistical analysis software package 'Statistical Package for the Social Sciences' (SPSS Inc, Chicago, IL, USA) 11.0 for Windows.

\section{RESULTS}

\section{NO is not essential for stomatal closure in Arabidopsis leaves under water-stress conditions}

As has been previously observed by others, with fully turgid leaves, the NO scavenger PTIO had a marked effect in inhibiting the stomatal closure normally induced by either ABA or SNP. Here, the mean apertures, after treatment

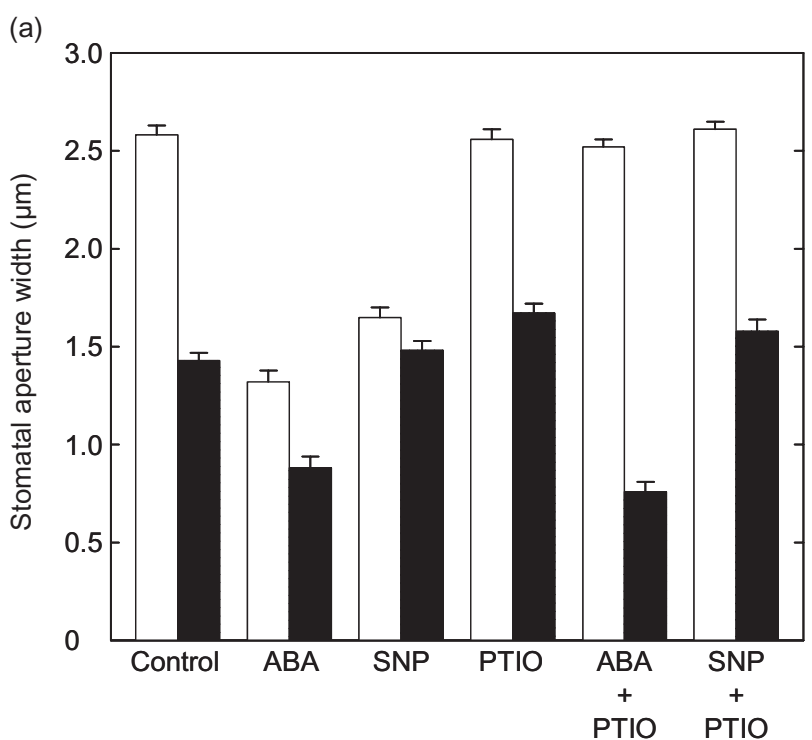

(b)

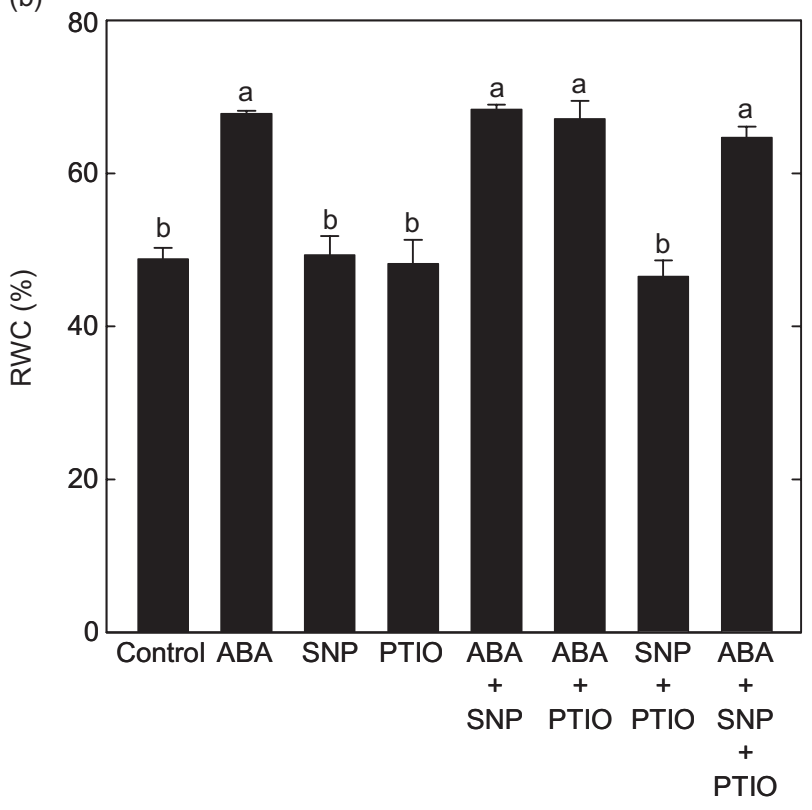


with these compounds, did not differ significantly $(P<0.05$ by $t$-test) from those of the control (Fig. 1a, white bars). Alone, PTIO had no significant effect on stomatal apertures when compared with those of the control leaves treated with buffer alone $(P<0.05$ by $t$-test $)$.

The pre-treatment of leaves with ABA, but not SNP, enhanced stomatal closure following dehydration stress (Fig. 1a, black bars). ABA-induced stomatal closure was not inhibited in dehydrated leaves treated with PTIO suggesting that $\mathrm{NO}$ was not required for ABA-induced stomatal closure under conditions of water shortage. Moreover, the stomatal apertures of SNP pre-treated dehydrated leaves were not significantly different from those of the untreated controls $(P<0.05$ by $t$-test $)$. Similarly, under conditions of water stress, treating the leaves with SNP + PTIO together had no significant effect on stomatal apertures $(P<0.05$ by $t$-test). Together, these data suggest that NO synthesis and action are not required during the ABA-induced stomatal closure observed in leaves deprived of adequate water. Concurring with these results, leaves treated with ABA or ABA + PTIO retained 28 and 29\%, respectively, more water than untreated controls following $2 \mathrm{~h}$ of water stress (Fig. 1b), while leaves treated with either SNP or SNP + PTIO did not exhibit significant differences in their RWC as compared with the controls $(P<0.05$ by Tukey test). When leaves were co-incubated with ABA + SNP, their RWC was not significantly altered from that of leaves treated with ABA alone, suggesting that the water-stress tolerance exhibited by ABA-treated leaves was not based on a NO-dependent mechanism (Fig. 1b). In alignment with these results, leaves treated with $\mathrm{ABA}+\mathrm{SNP}+\mathrm{PTIO}$ did not exhibit any significant difference in their RWC as compared with leaves treated with ABA alone. PTIO alone appeared to have no effect.

\section{NR mutations do not affect ABA-enhanced stomatal closure in leaves under water-stress conditions}

To discern whether or not an endogenous source of NO was required for ABA-induced stomatal closure, we employed the nia1 nia2 double mutant. As has been shown previously in epidermal peels (Desikan et al. 2002), here, both ABA and nitrite had little effect on the stomatal apertures of fully hydrated nia1 nia2 mutant leaves (Fig. 2a). In fully turgid leaves of wild-type plants, ABA and nitrite induced stomatal closure, causing a significant reduction in stomatal apertures $(P<0.05$ by $t$-test $)$. However, the stomatal apertures of turgid plants treated with either ABA $(50 \mu \mathrm{M})$ or nitrite ( $1 \mathrm{~mm}$ ) were found to be 64 and $81 \%$ greater, respectively, in leaves of the nial nia2 mutant than in those of wild-type plants. Also, the incubation of either wild-type or nial nia2 leaves in either $\mathrm{NaCl}$ (1 mM, data not shown) or nitrate ( $1 \mathrm{~mm}$; as a negative control for sodium nitrite) did not induce stomatal closure. To support the case that the insensitivity of nia1 nia2 stomata to ABA and nitrite resulted from the reduced ability of NR to generate NO in
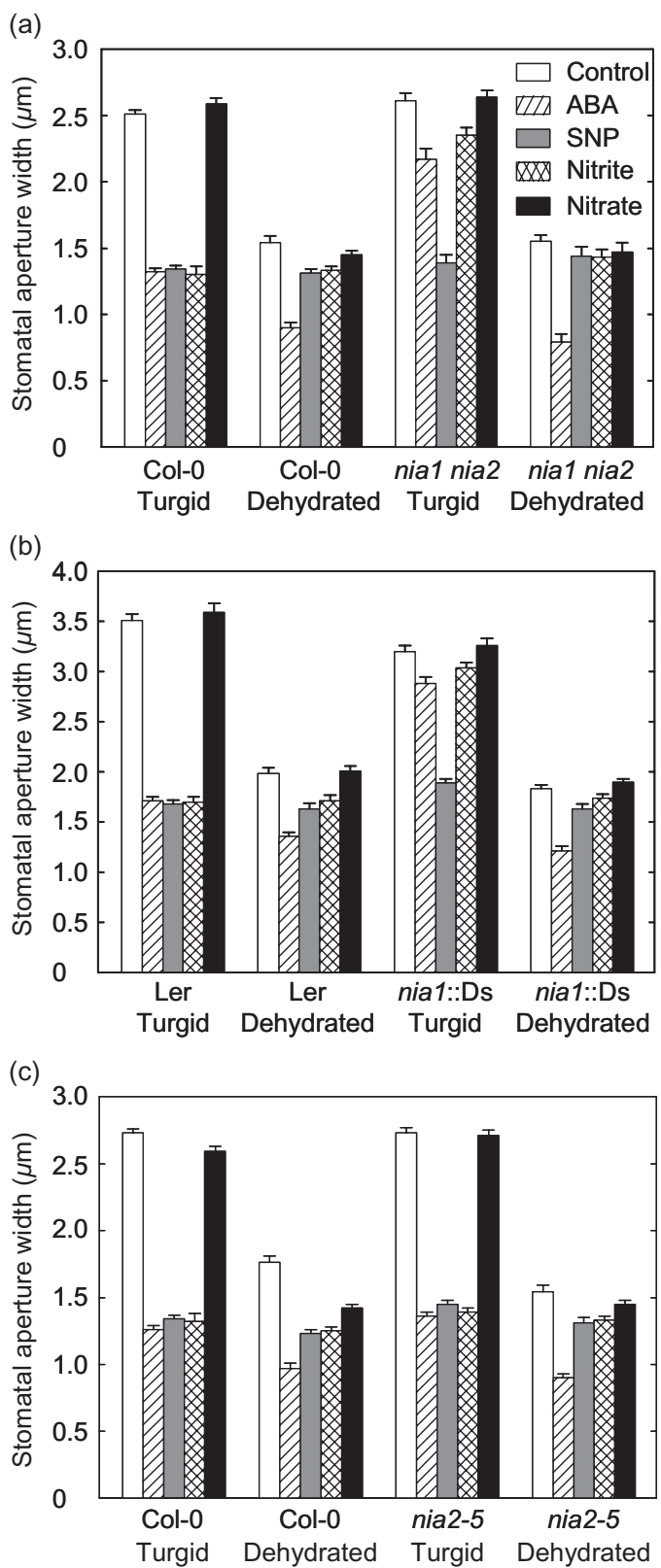

Figure 2. NR mutations do not affect abscisic acid (ABA)-enhanced stomatal closure in leaves under water-stress conditions. Leaves from either wild-type, nia1 nia2 [background Columbia (Col-0), (a)], nia1::Ds [background Landsberg erecta (Ler), (b)] or nia2-5 [background Col-0, (c)] plants were floated in 2-(N-morpholino)ethanesulfonic acid (MES)-KCl buffer under light to induce stomatal opening. Afterwards, leaves were incubated for $2.5 \mathrm{~h}$ in buffer alone (control), $50 \mu \mathrm{M} \mathrm{ABA,} 50 \mu \mathrm{M}$ sodium nitroprusside (SNP), $1 \mathrm{~mm}$ nitrite or $1 \mathrm{~mm}$ nitrate. Stomatal apertures were measured before dehydration and 30 min after submitting leaves to dehydration under a direct light source. The data are representative of four independent experiments with means of 100 stomata \pm standard errors.

these tissues, turgid leaves of the mutant were exposed to NO via their incubation with SNP. Here, the SNP caused stomatal closure in the well-hydrated leaves of both wildtype (Col-0) and nia1 nia2 mutant plants (Fig. 2a). 
Under dehydrating conditions, the stomata of both wildtype and nia1 nia2 plants exhibited similar increased closure following ABA treatment (Fig. 2a), again indicating that NO resulting from the activity of NR is not required for ABA-induced stomatal closure when water availability is limited. Additionally, following dehydration, no significant difference $(P<0.05$ by $t$-test $)$ in stomatal closure was observed between wild-type (Col-0) and nia1 nia2 leaves treated with nitrite, a known substrate of NO synthesis (Garcia-Mata \& Lamattina 2003; Neill et al. 2003). When the $\mathrm{NO}_{2}^{-}$was replaced by $\mathrm{NO}_{3}^{-}$, both nial nia2 and wildtype plants showed similar stomatal apertures (Fig. 2a). Also, nia1 nia2 and wild-type stomata did not display enhanced stomatal closure when dehydrated leaves were pre-exposed to NO via their incubation with SNP (Fig. 2a).

Further evidence that a loss of NR function suppresses stomatal closure induced by ABA in turgid, but not dehydrated leaves, was obtained using the two single NR mutants. Two genes encoding NR are present in the Arabidopsis genome, NIA1 and NIA2 (encoded proteins are often referred to as NR1 and NR2, respectively). In seedlings, NR2 accounts for $90 \%$ of the total NR-mediated nitrate to nitrite assimilatory activity, while NR1 accounts for the remaining 10\% (Wilkinson \& Crawford 1991). The two genes show a high degree of coding sequence similarity and the two isoforms are $83.5 \%$ identical at the amino acid level but do show some localized areas of sequence divergence in the first $90 \mathrm{~N}$-terminal amino acids and in various other regions (Wilkinson \& Crawford 1993). Both genes appear to be expressed in guard cells (Bright 2006).

To identify if mutations in the individual NR-encoding genes affected ABA responses in the guard cells of turgid and drought-stressed leaves, stomatal closure was compared between wild-type, nia1::Ds and nia2-5 plants following such treatments. In turgid leaves, ABA-induced stomatal closure was significantly impaired in the leaves of the nia1::Ds mutant compared with that observed in wellhydrated leaves of wild-type plants (Fig. 2b). Here, the ABA treatment of leaves resulted in only a $10 \%$ reduction in the stomatal apertures of the nia1::Ds mutant, while in leaves of wild-type (Ler) plants, stomatal apertures reduced by $50 \%$ (Fig. 2 b). In turgid leaves of wild-type plants, nitrite induced a $51 \%$ reduction in stomatal apertures but failed to induce any significant reduction in the stomatal apertures of similarly treated nial::Ds plants $(P<0.05$ by $t$-test $)$. Additionally, in turgid leaves, stomata of nia1::Ds plants responded to SNP treatment in the same manner as those of wild type (Fig. 2b). Thus, these data support the concept that NR1 function is required for ABA-mediated stomatal responses in turgid leaves.

We also investigated the effect of the mutation in NIAI on ABA-induced stomatal closure in dehydrated leaves. In this case, ABA-induced stomatal closure in dehydrated leaves was not affected by the loss of function of NR1 (Fig. 2b). After pre-treatment with ABA, there was no significant difference $(P<0.05$ by $t$-test) between the reduction of stomatal apertures in wild-type and nia1::Ds leaves, suggesting that under these dehydrated conditions, the
ABA-enhanced response of guard cells occurs via an NR1-independent mechanism. Similarly, nitrite-enhanced stomatal closure was abolished in the dehydrated leaves of wild-type plants in that the mean stomatal aperture of treated leaves did not differ significantly from those of control leaves $(P<0.05$ by $t$-test $)$. Also, SNP pre-treatment failed to enhance stomatal closure in the dehydrated leaves of both nia1::Ds and wild-type plants (Fig. 2b). Together, these data indicate that functional NR1 is not required for ABA-mediated stomatal closure in dehydrated leaves.

On the other hand, in turgid leaves of nia2-5 plants, both ABA and nitrite induced significant stomatal closure in comparison with that observed in untreated control leaves $(P<0.05$ by $t$-test; Fig. 2 c). nia2-5 stomata also responded to ABA, SNP and nitrite in a similar manner to those of wildtype plants. These data suggest that NR2 function is not required for guard cell responses to $\mathrm{ABA}$ in fully turgid leaves.

As for both wild-type and nia1::Ds plants, under dehydrating conditions, the pre-treatment of nia2-5 plants with either SNP or nitrite did not significantly further enhance stomatal closure. However, as for wild-type, nial::Ds and nia1 nia2 plants, stomatal closure was enhanced by the ABA treatment of nia2-5 plants subsequently subjected to dehydration (Fig. 2c). Thus, together, the data suggest that functional NR may not be required for ABA-induced stomatal closure in Arabidopsis leaves subjected to conditions of water stress that lead to dehydration.

As such, it then appears that, of the two NR isoforms, NR1 alone is functionally involved in ABA-induced stomatal closure and this only when plants are well hydrated and the leaves fully turgid. To ascertain that NR exerts its influence on guard cell movements via NO synthesis, we performed a series of DAF2-DA fluorescence confocal microscopy investigations using wild-type and NR mutant plants subjected to a variety of treatments. The treatment of turgid leaves of nial::Ds plants with ABA did not induce increased NO synthesis in their stomatal guard cells, as it did in those of wild type $(P>0.05$, Fig. 3a). Similarly, the treatment of nia1::Ds leaves with nitrite failed to enhance significantly the increased level of guard cell DAF2-DA fluorescence similar to that seen in wild-type leaves (Fig. 3a). In fact, in the presence of nitrite, nial::Ds guard cell DAF2 fluorescence appeared significantly reduced $(P<0.01)$ compared with the untreated controls.

In fully turgid leaves, apparent guard cell NO synthesis (as seen via DAF2 fluorescence) in response to ABA was marginally but significantly $(P<0.01)$ reduced in the nia2-5 plants by $12 \%$, compared with those of wild-type plants (Fig. 3b). However, in the nia2-5 plants, the increase in guard cell DAF-2DA fluorescence stimulated by nitrite was not significantly different from that observed in similarly treated wild-type plants $(P>0.05$, Fig. 3b). These data support the idea that it is NR1 and not NR2 that is essential for ABA-induced NO synthesis in the guard cells of turgid leaves and that this is required for stomatal closure to occur. 
(a)

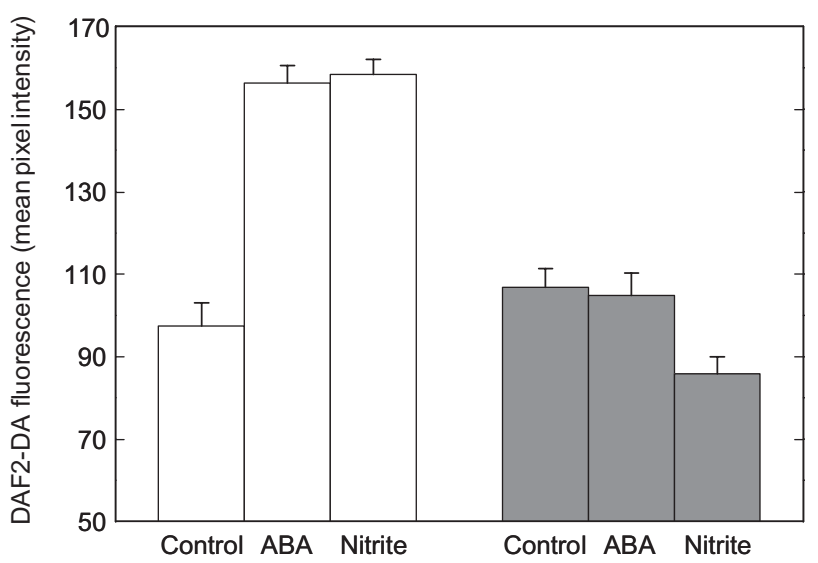

(b)

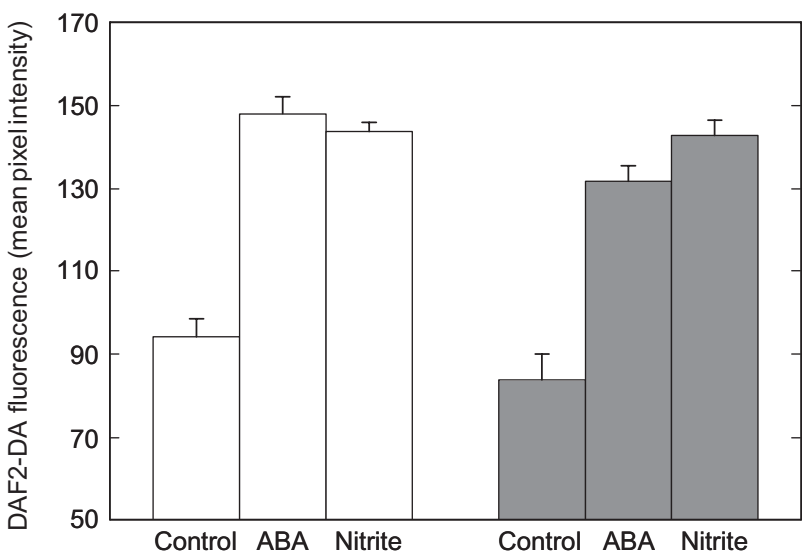

Figure 3. Nitrate reductase (NR)1, but not NR2 is required for abscisic acid (ABA)- and nitrite-induced guard cell nitric oxide (NO) generation. ABA and nitrite do not induce NO synthesis in nia1::Ds guard cells (a), but do in those of nia2-5 plants (b). Wild-type Columbia, Landsberg erecta (white bars) and nia1::Ds, nia2-5 (grey bars) Arabidopsis leaf epidermal fragments were incubated with 4,5-diaminofluorescein diacetate (DAF2-DA) in 2-(N-morpholino)ethanesulfonic acid (MES)-KCl buffer. Fragments were treated with ABA $(10 \mu \mathrm{M})$ or nitrite $(1 \mathrm{mM})$ for $25 \mathrm{~min}$. Confocal microscopy data are displayed as mean pixel intensities of nine independent biological replicates \pm standard errors.

\section{SNP does not induce stomatal closure in leaves from aba1-1 and abi1-1 plants}

To investigate the role of endogenous ABA and ABA signalling in NO-induced stomatal closure, we made use of ABA deficient (abal-1) and ABA insensitive (abi-1) mutants. As expected, the stomata of well-watered abal-1 plants closed to a lesser degree, but in similar fashion to those of wild-type plants following ABA treatment (Fig. 4a). However, while the treatment of fully turgid abal-1 leaves with SNP did reduce stomatal apertures, this was to a markedly reduced extent in comparison with that observed to occur in the leaves of similarly treated wildtype leaves (Fig. 4a). As with wild-type plants, ABA, but not SNP, appeared to enhance the closure of stomata in the leaves of dehydrated aba1-1 plants (Fig. 4a). Under wellwatered conditions, stomata of the ABA-insensitive abil-1 mutant failed to close in response to treatment with either ABA or SNP, even when these compounds were used at concentrations that induced near to complete closure of the stomata in the turgid leaves of wild-type plants (Fig. 4b). Both these treatments also failed to induce stomatal closure in the leaves of dehydrated abil-1 plants. These data indicate that the ability of NO to exert its effects on the movement of the guard cells of well-hydrated leaves depends on the presence of endogenous ABA and its ability to either signal downstream of ABI1 or to engage the ABAsignalling pathway to some extent. Put in another way, it appears that, under hydrated conditions, for NO to be

(a)

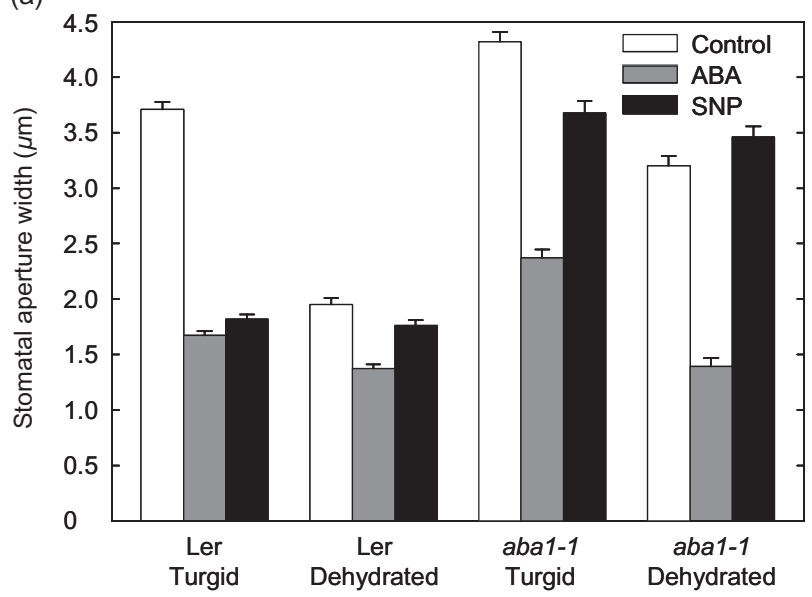

(b)

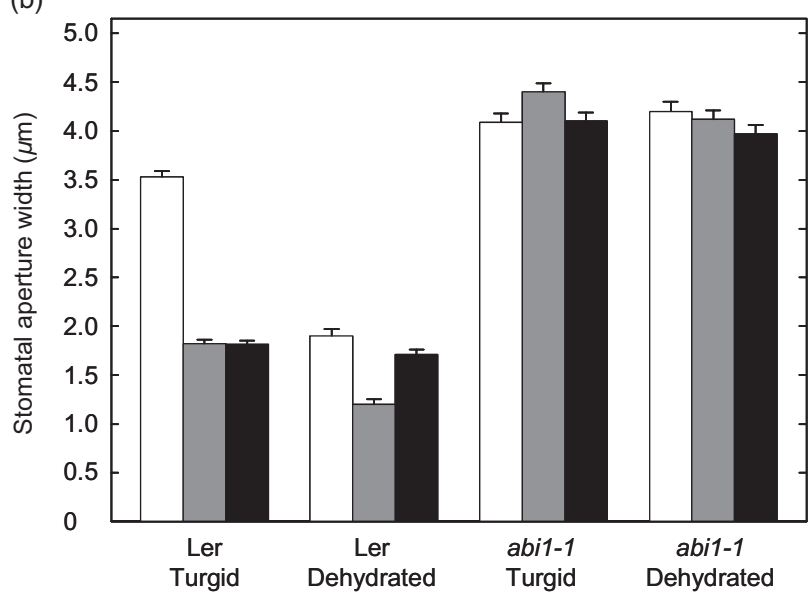

Figure 4. Nitric oxide (NO) does not induce stomatal closure in both turgid leaves and water-stressed leaves from $A B A$ deficient 1 (aba1-1) (a) and ABA insensitive 1 (abi1-1) (b) plants. Leaves from wild-type [Landsberg erecta (Ler)], abal-1 and abil-1 plants were floated in 2-(N-morpholino)ethanesulfonic acid (MES)-KCl buffer under light to induce stomatal opening and then incubated for $2.5 \mathrm{~h}$ in buffer alone (control), $50 \mu \mathrm{M}$ abscisic acid (ABA) or $50 \mu \mathrm{M}$ sodium nitroprusside (SNP). Stomatal apertures were measured before dehydration and $30 \mathrm{~min}$ after submitting the leaves to dehydration under a direct light source. The data are representative of four independent experiments with means of 100 stomata \pm standard errors. 
active in guard cells, the ABA-signalling pathway must be operative and at least signalling the presence of some ABA, even if this is insufficient to cause full stomatal closure.

\section{ABA-enhanced water-stress tolerance does not require functional NO signalling}

To determine whether or not NO-determined stomatal apertures influence plant water loss, leaves of nial nia2, nia1::Ds, nia2-5 and wild-type plants were treated with ABA, SNP or nitrite and short-term water-loss assays were performed by evaluating their decline in $\mathrm{FW}$ with time.

ABA treatment reduced the water loss of detached wild-type leaves by approximately a third after $3 \mathrm{~h}$ under dehydrating conditions, while SNP treatment did not significantly diminish either the rate or amount of water lost compared with that lost by untreated control leaves (Fig. 5). Here, leaves of nia1 nia2, nia1::Ds and nia2-5 plants exhibited similar levels of ABA-induced tolerance to water stress as observed in those of wild-type plants. Conversely, leaves of the ABA-insensitive signalling mutant, abi1-1, failed to show improved water retention after ABA treatment and, again, this position was not recovered by SNP treatment (Fig. 5). Thus, the implication is that, in Arabidopsis, it is ABA that is primarily responsible for water conservation during the sudden application of conditions of water stress and that NO signalling is not necessarily a critical component of this short-term ABA-induced ability to conserve water in response to conditions of drought.

\section{NO does not enhance water-stress tolerance in intact Arabidopsis plants}

The above data suggest that NO is not required for the ABA-induced stomatal closure and associated water conservation that occurs during the rapid dehydration in detached leaves. However, it has been suggested that detached-leaf water-loss assays are insufficiently sensitive to detect small variations in Arabidopsis leaf water loss which may continue to occur after long periods of water stress (Verslues et al. 2006). Therefore, we also generated water loss data using whole plants. Figure $6 a$ shows that under well-watered conditions at time zero, there was no significant difference $(P<0.05$ by Tukey test $)$ in the RWC of wild-type plants and either nia2-5 or nia1::Ds plants treated with buffer (Control), ABA or SNP. When the plants were submitted to a long period (14 d) of drought, wild-type and nia2-5 plants treated with ABA retained approximately 45 and $50 \%$ more water, respectively, than untreated control plants. Conversely, the RWC of wild-type and nia2-5 plants treated with SNP were reduced in a similar way to wild-type plants $(P<0.05$ by Tukey test $)$. Similar data were also determined for nial::Ds plants. After $14 \mathrm{~d}$ of water deprivation, ABA-treated wild-type and nia1::Ds plants retained approximately 27 and $38 \%$ more water, respectively, than the control plants (Fig. 6a). Again, the RWC of wild-type

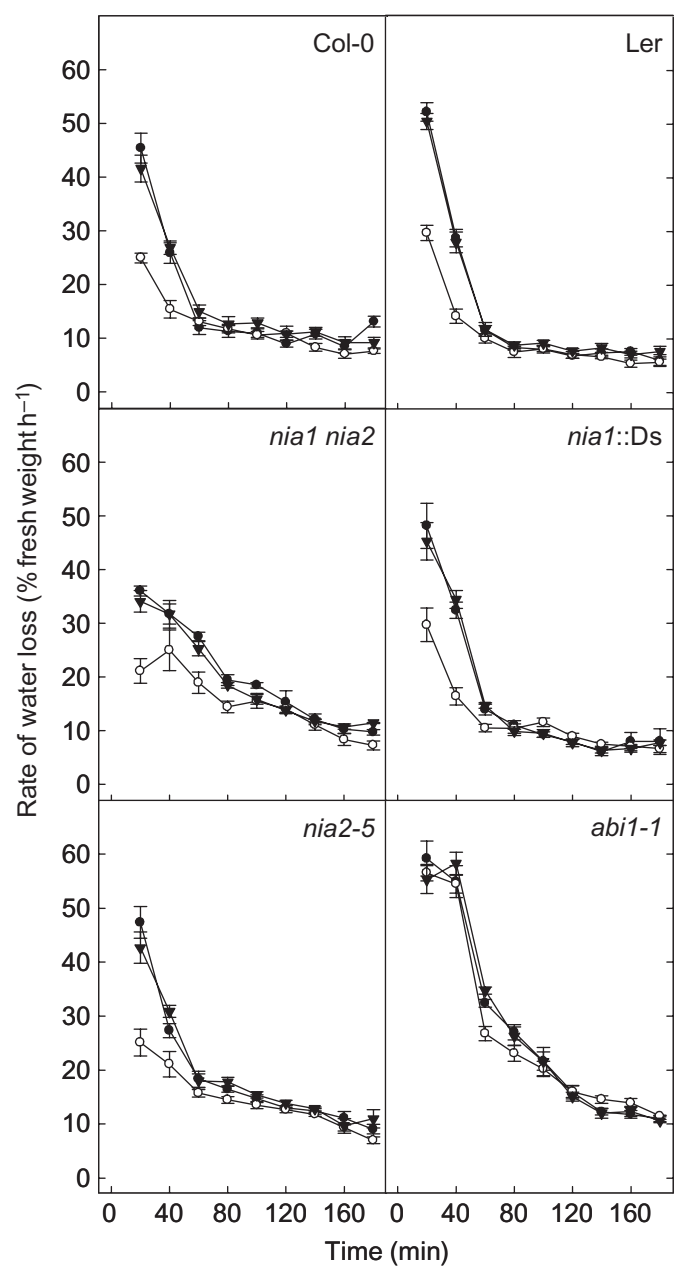

Figure 5. Unlike abscisic acid (ABA), nitric oxide does not diminish water loss under conditions of water stress. Leaves of either wild-type [Columbia (Col-0) and Landsberg erecta (Ler)], nia1 nia2, nia2-5, nia1::Ds and ABA insensitive 1 (abi1-1) plants were floated in 2-(N-morpholino)ethanesulfonic acid (MES)-KCl buffer under light to induce stomatal opening. Afterwards, leaves of wild-type and mutant plants were incubated for $2.5 \mathrm{~h}$ in either buffer alone (control, black circle), $50 \mu \mathrm{M} \mathrm{ABA} \mathrm{(white} \mathrm{circle)} \mathrm{or}$ $50 \mu \mathrm{m}$ sodium nitroprusside (black triangle). Leaves were then removed from solution, gently dried with absorbent tissue and were incubated under a direct light source. The percentage loss of fresh weight with time was used as a measure to indicate water lost and the rate of water loss was then determined accordingly for each plant type with each treatment. Data are derived from two independent experiments with four replicates of four leaves each for each treatment \pm standard errors.

and nial::Ds plants treated with SNP were similar to the controls.

\section{NO does not enhance stomatal closure in whole Arabidopsis plants under conditions of drought}

Under well-watered conditions, stomatal closure was induced to a similar degree in nia2-5 and wild-type plants 

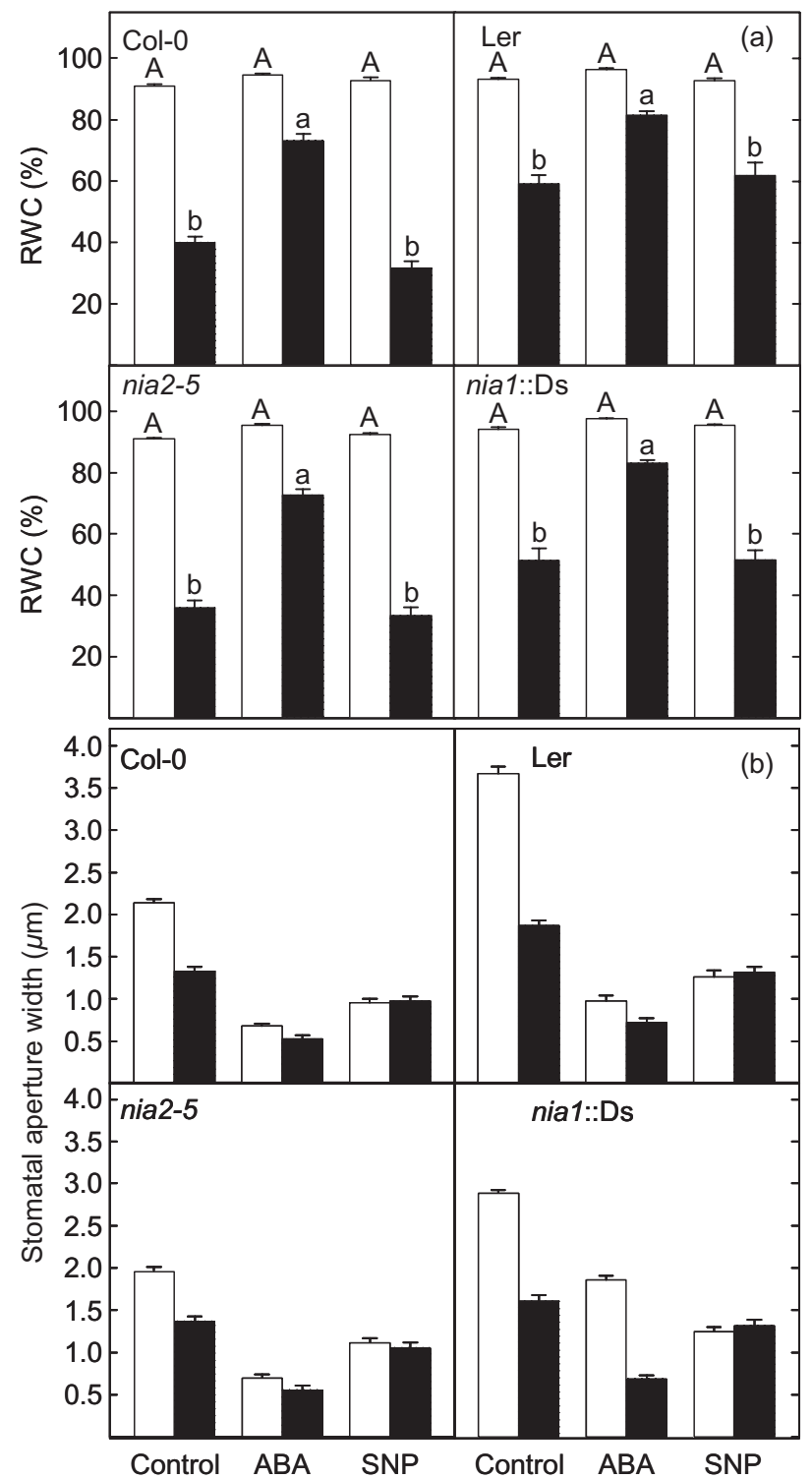

Figure 6. Unlike abscisic acid (ABA), nitric oxide does not enhance drought tolerance by enhancing stomatal closure during periods of water deprivation. The aerial parts of 4-week-old nia1::Ds, nia2-5 and wild-type Arabidopsis plants were pre-treated by their submergence in either buffer alone or solutions of ABA $(50 \mu \mathrm{M})$ or sodium nitroprusside (SNP) $(50 \mu \mathrm{M})$ for $5 \mathrm{~s}$ each day for $3 \mathrm{~d}$ prior to their subsequent exposure to drought stress conditions. Drought was induced by withholding the watering of plants maintained in the growth chamber. Ten leaves from four different plants were removed before the start of the drought treatment (white bars) and after $14 \mathrm{~d}$ of drought (black bars) and their relative water contents (RWC) (a) determined. Stomatal apertures (b) were measured in Arabidopsis leaves immediately before the start of drought stress (white bars) and $7 \mathrm{~d}$ after the start of drought stress (black bars). The data are representative of three independent experiments with the means of 120 stomata \pm standard errors. following their periodic treatment with either ABA or SNP over a 3 day time course (Fig. 6b). After the same pretreatment time course and under similar well-watered conditions, ABA-mediated stomatal closure was significantly reduced in nia1::Ds plants compared with the relevant wild-type controls (Fig. 6b). However, the nial::Ds guard cells of well-hydrated plants responded to SNP in the same manner as those of wild-type plants. Thus, these data confirm a requirement for NR1, but not NR2, in mediating guard cell responses to ABA in plants under well-watered conditions. Nevertheless, although the movement of turgid nia1::Ds guard cells was much reduced, there was a degree of stomatal closure in response to protracted ABA treatment, suggesting that some stomatal closure independent of NR1-generated $\mathrm{NO}$ can occur in well-watered plants exposed to ABA for extended periods.

Following $7 \mathrm{~d}$ of drought stress, applied after $3 \mathrm{~d}$ of ABA pre-treatment, ABA-induced stomatal closure was restored to near wild-type levels $(P<0.05$ by $t$-test $)$ in nial::Ds plants (Fig. 6b). Again, these data suggest that a functional NR1 protein was not a requirement of stomatal closure under conditions of water deprivation. Indeed, in comparison with either buffer only or SNP-treated plants, the pretreatment of wild-type, nia1::Ds or nia2-5 plants with ABA significantly enhanced the degree of stomatal closure that occurred after the subsequent drought period (Fig. 6b). In contrast, the 3 day pre-treatment of wild-type, nia1::Ds or nia2-5 plants with SNP did not enhance the extent of postdrought stomatal closure beyond that seen in similarly treated well-watered plants.

Thus, the enhanced reduction in drought-related water loss exhibited by the ABA pre-treated plants (Fig. 6a) can likely be attributed to an NO-independent effect of ABA that results in reduced transpiration as a consequence of enhanced stomatal closure. Taken together, the data suggest that here, NO was not an essential component of the ABAinduced stomatal closure that occurred when the plants were exposed to conditions that resulted in prolonged drought stress.

\section{NO is required for guard cell movements during the light-to-dark transition in turgid leaves}

Stomatal guard cells generate NO when challenged with ABA. However, the inference from the above data is that this NO synthesis is not required for the stomatal closure that occurs in response to dehydration. Thus, in order to demonstrate a physiological role for NO in the movement of the guard cells of well-hydrated leaves, we examined its role during the stomatal closure that occurs during the light-to-dark transition. $\mathrm{NO}$ is known to accumulate in the guard cells of leaves when they are placed in the dark and this has been correlated with the stomatal closure that occurs (She, Song \& He 2004). Here, when well-hydrated wild-type leaves were placed in the dark for $2 \mathrm{~h}$, their stomata closed (Fig. 7a) and this correlated with increased guard cell DAF2-DA fluorescence (Fig. 7b), indicating that 
(a)

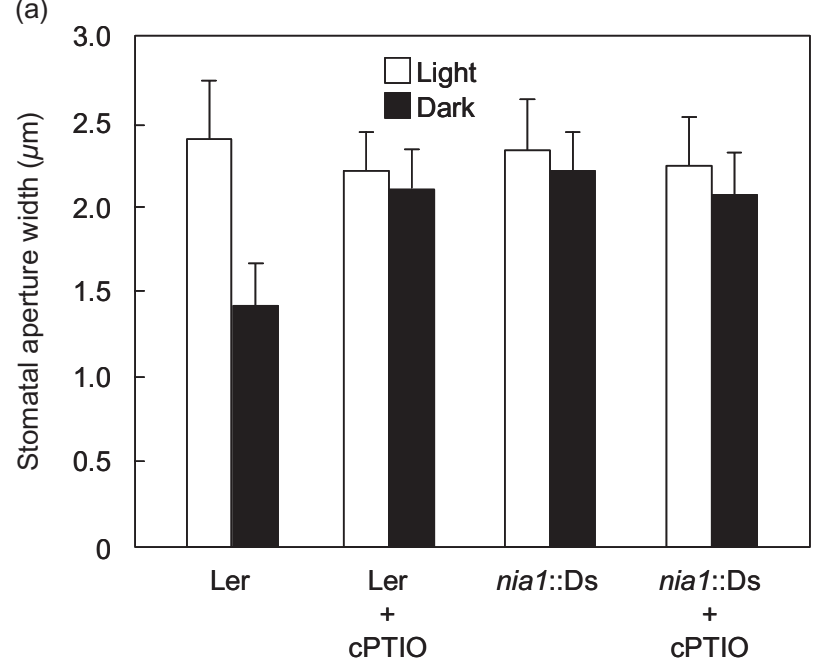

(b)

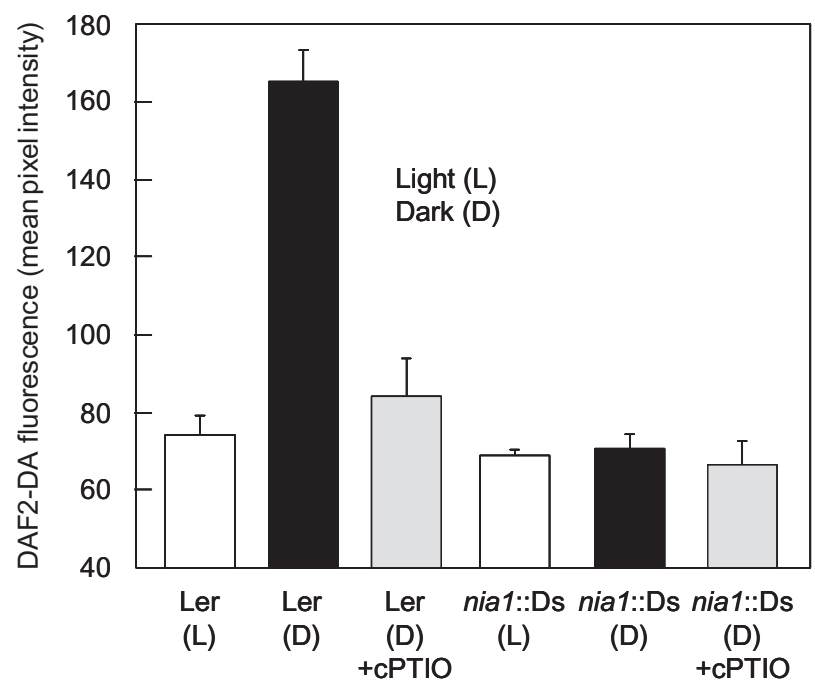

Figure 7. Guard cell nitric oxide (NO) synthesis is required for dark-induced stomatal closure. Leaves of either wild-type [Landsberg erecta (Ler)] or nia1::Ds plants were floated on 2-(N-morpholino)ethanesulfonic acid (MES)- $\mathrm{KCl}$ buffer with and without $200 \mu \mathrm{m} 2$-(4-carboxyphenyl)-4,4,5,5-

tetramethylimidazoline-1-oxyl-3-oxide (cPTIO) under

illumination to induce stomatal opening and were then either maintained in the light (white bars) or transferred to the dark (black bars) for $2 \mathrm{~h}$. After incubation, stomatal apertures were determined (a). Guard cell NO-associated 4,5-diaminofluorescein diacetate (DAF2-DA) fluorescence in epidermal fragments of wild-type and nia1::Ds leaves was examined by laser scanning confocal microscopy after $2 \mathrm{~h}$ in either the light or dark with or without removal of endogenous NO by cPTIO (b). The data are representative of two independent experiments with means of 50 stomata \pm standard errors.

increased synthesis of NO had occurred. Removal of the NO by treating the leaves with cPTIO effectively prevented this dark-induced stomatal closure (Fig. 7a) and the associated DAF-2DA fluorescence (Fig. 7b). When well-hydrated wild-type leaves were transferred from the dark to the light, their stomata reopened (Fig. 8). This light-induced reopening was inhibited by both ABA and SNP (Fig. 8). The co-incubation of dark-treated leaves with ABA and cPTIO significantly reduced the inhibitory effect of the ABA on the light-induced stomatal reopening (Fig. 8). While the stomata in the leaves of nia2-5 plants behaved as those of wild-type plants (data not shown), those of nial::Ds plants failed to close when transferred to the dark (Fig. 7a) and their guard cells showed considerably reduced DAF2-DA fluorescence during this treatment when compared with those of similarly treated wild-type plants (Fig. 7b). Thus, it can be concluded that dark-induced stomatal closure in well-hydrated leaves requires $\mathrm{NO}$ synthesis and action and that removal of the NO on the return to the light is required for stomatal reopening.

\section{DISCUSSION}

\section{NO is not required for ABA-induced stomatal closure during drought}

NO has been implicated as a key signalling molecule during responses to various abiotic and biotic stresses (Beligni \& Lamattina 2001; Arasimowicz \& Floryszak-Wieczorek 2007; Neill et al. 2008) and in the ABA signal transduction pathway which determines stomatal apertures (GarciaMata \& Lamattina 2002; Neill et al. 2002; Wilson, Neill \& Hancock 2008). Previous work with well-hydrated Arabidopsis plants has demonstrated that ABA-induced stomatal closure is dependent on NR-mediated NO synthesis

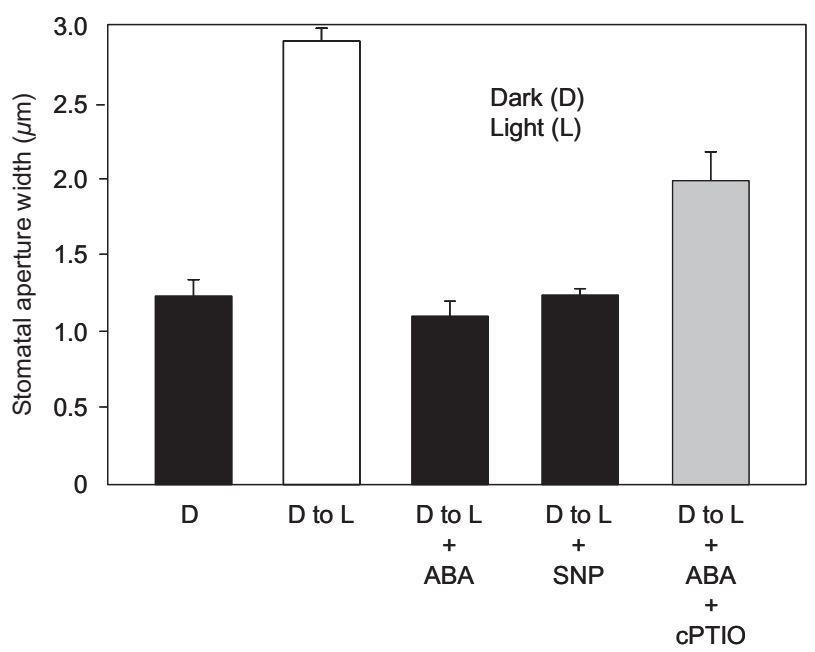

Figure 8. Guard cell abscisic acid (ABA)-induced NO accumulation prevents light-induced stomatal reopening. Wild-type [Landsberg erecta (Ler)] leaves were floated on 2-(N-morpholino)ethanesulfonic acid (MES)-KCl buffer for $2 \mathrm{~h}$ in the dark to close their stomata, at which point, they were returned to the light for a further $2 \mathrm{~h}$ and either left untreated or treated with $50 \mu \mathrm{m} \mathrm{ABA}, 50 \mu \mathrm{m}$ sodium nitroprusside (SNP) and $50 \mu \mathrm{M} \mathrm{ABA}+200 \mu \mathrm{M}$ 2-(4-carboxyphenyl)-4,4,5,5tetramethylimidazoline-1-oxyl-3-oxide (cPTIO). Stomatal apertures were then measured. The data are representative of two independent experiments with means of 100 stomata \pm standard errors. 
(Desikan et al. 2002). The data described here demonstrate that stomatal responses to NO differ between turgid and dehydrated tissues. The treatment of fully turgid leaves of Arabidopsis with the NO scavenger PTIO largely suppressed stomatal responses to ABA and SNP (Fig. 1a). Zhang et al. (2007a) have shown that water stress, induced by polyethylene glycol treatment, stimulates NO production in leaves as a result of increased ABA synthesis. However, our data show that ABA-induced stomatal closure was not suppressed by the removal of NO in waterstressed leaves. In contrast to the obvious effect seen with ABA, the treatment of leaves with SNP appeared to do little in terms of maintaining higher RWC under dehydrating conditions (Fig. 1b). Furthermore, removal of the NO with PTIO failed to prevent the ABA-mediated decrease in transpiration that occurred under these conditions.

\section{The requirement for guard cell NR1-mediated NO signalling is bypassed during drought}

The genetic evidence indicates that, in the guard cells of ABA-treated turgid leaves, $\mathrm{NO}$ is generated by NR (Desikan et al. 2002). Guard cells of the NR-deficient Arabidopsis double mutant, nia1 nia2 (Wilkinson \& Crawford 1993), fail to accumulate NO under conditions that normally stimulate its synthesis (Desikan et al. 2002). Both NIA1 and NIA2 are expressed in Arabidopsis guard cells (Bright 2006) and NR has been shown to convert nitrite to NO (Dean \& Harper 1986; Rockel et al. 2002; Bright et al. 2006). Confirming previous observations, while neither ABA nor nitrite induced stomatal closure in the fully turgid leaves of nia1 nia2 plants, stomatal closure occurred after their treatment with SNP (Fig. 2a), implying that NR-mediated NO synthesis is required for ABA-induced stomatal closure under well-watered conditions. However, nia1 nia2 plants are not obviously wilty and, therefore, must be able to regulate their transpiration under conditions of drought. Indeed, as in wild-type plants, the application of ABA significantly enhanced stomatal closure in the leaves of this mutant under conditions of water deprivation. Importantly, under these conditions, the treatment of both wild-type and nial nia2 plants with SNP did not enhance stomatal closure (Fig. 2a).

Our data show that it is specifically the guard cells of turgid leaves of nia1::Ds (Fig. 3a), but not nia2-5 (Fig. 3b) plants, which are unable to synthesize NO in response to either ABA or nitrite and that, as a consequence, the stomata of nia1::Ds plants fail to close in response to such treatments (Fig. 2b) but close in response to SNP treatment. The treatment of fully turgid nia2-5 leaves with ABA, nitrite or SNP results in stomatal closure similar to that which occurs in wild-type plants (Fig. 2c). Thus, we would concur with the concept that, of the two isoforms, it is specifically NR1 that plays a role in NO signalling. As with nial nia2 plants, under water-stress conditions, ABA-induced stomatal closure was not abolished in the nia1::Ds mutant, suggesting that functional NR1 is not required for ABAmediated stomatal closure in dehydrated leaves (Fig. 2b).
Thus, stomatal closure in water-stressed leaves most likely results from ABA signalling that bypasses NR1-mediated NO signalling.

\section{Guard cell NR1-mediated NO signalling may operate via the $\mathrm{Ca}^{2+}$-dependent ABA-signalling pathway}

It is interesting that stomatal responses to $\mathrm{NO}$ appear to require the presence of a functional ABA-signalling pathway. Fully turgid guard cells of the ABA-insensitive mutant, abi1-1, accumulate NO in response to ABA treatment, but their stomata remain open (Desikan et al. 2002). We have also shown that SNP also fails to induce stomatal closure in the fully hydrated leaves of this mutant (Fig. 4b). Similarly, although stomata of well-hydrated leaves of the ABA-deficient mutant aba1-1 closed in response to ABA, their response to SNP was substantially reduced. Thus, it would appear that, in order for guard cell responses to NO to occur, not only is a functional ABA-signalling pathway required but also some degree of its activation. It is noticeable that the stomatal apertures of the ABA-insensitive and ABA-deficient mutants are always considerably greater than those of wild-type plants grown under similar wellwatered conditions. This in itself indicates that in the turgid leaves of wild-type plants, the ABA-signalling pathway is always active to some extent. Presumably, the NO synthesized as a result of various environmental signals further mediates the activity of this pathway, so determining actual stomatal apertures.

The concept that both NO-dependent and NOindependent ABA-signalling pathways operate in guard cells parallels observations concerning the involvement of $\mathrm{Ca}^{2+}$ in ABA signalling. In turgid plants, ABA-dependent, guard cell NO signalling is considered to operate via mechanisms that elevate the level of intracellular $\mathrm{Ca}^{2+}$ ions which in turn affect the activity of $\mathrm{Ca}^{2+}$-dependent $\mathrm{K}^{+}$and $\mathrm{Cl}^{-}$ion channels (Garcia-Mata et al. 2003). NO is known to affect $\mathrm{Ca}^{2+}$ mobilization (Lamotte et al. 2006), and a requirement for altered $\mathrm{Ca}^{2+}$ flux in order for normal NO responses to occur in guard cells has been demonstrated in Vicia faba (Sokolovski et al. 2005). ABA is known to signal via both $\mathrm{Ca}^{2+}$-dependent (Grabov \& Blatt 1999) and $\mathrm{pH}$ dependent (Grabov \& Blatt 1997), but $\mathrm{Ca}^{2+}$-independent pathways (Allan et al. 1994; Romano et al. 2000; Outlaw 2003; Israelsson et al. 2006). The observation that NO accumulates in the guard cells of the abi1-1 mutant in response to ABA treatment but fails to cause stomatal closure is then consistent with the idea that NO signals via the $\mathrm{Ca}^{2+}$ dependent ABA-signalling pathway which is impaired in this mutant (Armstrong et al. 1995). Exactly how NO signals via this pathway is still unknown, but there is some suggestion that this may involve the S-nitrosylation of $\mathrm{Ca}^{2+}$ dependent $\mathrm{K}^{+}$channels (Sokolovski \& Blatt 2004). Presumably, the $\mathrm{Ca}^{2+}$-independent ABA-signalling pathway is able to bypass the need for NR1-mediated NO signalling during periods that potentially lead to rapid dehydration. 


\section{Role of NO signalling in the guard cells of turgid plants}

Thus, the question remains as to what the physiological role of NO is in determining stomatal apertures in turgid leaves. The observations of She et al. (2004) that guard cells accumulate NO in the dark offered one avenue of investigation, and the data presented here indicate that NR1-mediated $\mathrm{NO}$ generation is required for the stomatal closure that occurs during the light-to-dark transition. Here, the removal of the ability of ABA to prevent the light-induced reopening of stomata by the NO scavenger cPTIO (Fig. 8) and the failure of nia1::Ds stomata to close in the dark coincident with a lack of NO synthesis in their guard cells provide compelling evidence that increased NO accumulation and signalling is a requirement during this event. Other recent studies also indicate that NO inhibits the lightinduced reopening of stomata and that this involves the ABA-signalling pathway (Yan et al. 2007; Zhang et al. 2007b) and downstream $\mathrm{Ca}^{2+}$ signalling (Garcia-Mata \& Lamattina 2007; Shimazaki et al. 2007). However, in many species, stomata do not always close in the dark and a multitude of other physiological and environmental factors determine whether or not this occurs (Caird, Richards \& Donovan 2007). The treatment of leaves of Pisum sativum with high levels of bicarbonate, for example, results in the accumulation of NO in their guard cells during the resulting stomatal closure (Kolla \& Raghavendra 2007). Thus, it is evident that a considerable degree of signalling crosstalk occurs in determining the stomatal apertures of turgid plants, and unravelling the full extent of the involvement of $\mathrm{NO}$ in this will require further investigation.

\section{Long-term effects of NO during periods of drought}

Despite having no immediately apparent role in the stomatal closure that occurs during the rapid onset of drought conditions, the exposure of leaves to NO may have other longer-term benefits. Various studies indicate that the prior exposure of plants to NO may be ameliorative during their subsequent exposure to drought (Garcia-Mata \& Lamattina 2001; Tian \& Lei 2006; Zhang et al. 2007a). However, the data presented here would argue that, under the dehydrating conditions applied, NO does not appear to enable enhanced water retention during drought stress. This observation is unlikely to have arisen as a consequence of the lower dose of the NO donor employed here as SNP concentrations between 10 and $150 \mu \mathrm{M}$ were similarly ineffective (data not shown). It is possible that the duration of the prior exposure of leaves to SNP and the rapidity of the application and duration of the subsequent stress period are factors determining the observed efficacy of NO in preventing water loss during periods of drought. Differences in the mode of application of the NO donor, that is, soil versus foliar application, may also account for some of the discrepancies between the observations of others and the data presented here. Alternatively, it may simply be that there exists some species-dependent variation in the long-term protection to dehydration offered as a result of exposure to elevated levels of NO.

\section{CONCLUSION}

In conclusion, the guard cell movements of turgid leaves are governed by a signalling network (Hetherington \& Woodward 2003; Fan, Zhao \& Assmann 2004) that has evolved to mediate the combined effects of multiple input signals and which may determine stomatal apertures by altering the activity of the ABA-signalling pathway. NR1-mediated NO signalling appears to be involved in mediating the movements of guard cells in the leaves of plants growing under well-watered conditions, possibly by altering the activity of components of the $\mathrm{Ca}^{2+}$-dependent ABAsignalling pathway. However, such NO signalling appears unlikely to be involved in the mechanisms that result in stomatal closure during the rapid onset of conditions of drought. A concerted systems approach will therefore be required to understand the role played by $\mathrm{NO}$ in mediating the movements of stomatal guard cells that occur in wellhydrated leaves in response to the numerous environmental stimuli known to affect this process.

\section{ACKNOWLEDGMENTS}

We are indebted to the Brazilian Council for the Advancement of Science and Technology $(\mathrm{CNPq})$ for the scholarship awarded to D.M.R. and for their financial support granted during the conduct of this research. A.C. was funded by a Portuguese government grant from the Fundação para a Ciência e a Tecnologia (FCT). R.D. was funded by an early career development fellowship from the Leverhulme Trust, UK. J.H. was funded by the Biotechnology and Biological Sciences Research Council, UK.

\section{REFERENCES}

Allan A.C., Fricker M.D., Ward J.L., Beale M.H. \& Trewavas A.J. (1994) Two transduction pathways mediate rapid effects of abscisic acid in Commelina guard cells. The Plant Cell 6, 1319-1328.

Arasimowicz M. \& Floryszak-Wieczorek J. (2007) Nitric oxide as a bioactive signaling molecule in plant stress response. Plant Science 172, 876-887.

Armstrong F., Leung J., Grabov A., Brearley J., Giraudat J. \& Blatt M.R. (1995) Sensitivity to abscisic acid of guard-cell $\mathrm{K}^{+}$channels is suppressed by abi1-1, a mutant Arabidopsis gene encoding a putative protein phosphatase. Proceedings of the National Academy of Sciences of the United States of America 92, 95209524.

Beligni M.V. \& Lamattina L. (2001) Nitric oxide in plants: the history is just beginning. Plant, Cell \& Environment 24, 267-278.

Bright J. (2006) Nitric oxide signalling in Arabidopsis thaliana guard cells. PhD Thesis, University of the West of England, Bristol, UK, pp. 132-377.

Bright J., Desikan R., Hancock J.T. \& Neill S.J. (2006) ABA-induced $\mathrm{NO}$ generation and stomatal closure in Arabidopsis are dependent on $\mathrm{H}_{2} \mathrm{O}_{2}$ synthesis. The Plant Journal 45, 113122. 
Caird M.A., Richards J.H. \& Donovan L.A. (2007) Night-time stomatal conductance and transpiration in $\mathrm{C}_{3}$ and $\mathrm{C}_{4}$ plants. Plant Physiology 143, 4-10.

Dean J.V. \& Harper J.E. (1986) Nitric oxide and nitrous oxide production by soybean and winged bean during the in vivo nitrate reductase assay. Plant Physiology 82, 718-723.

Desikan R., Griffiths R., Hancock J.T. \& Neill S.J. (2002) A new role for an old enzyme: nitrate reductase-mediated nitric oxide generation is required for abscisic acid-induced stomatal closure in Arabidopsis thaliana. Proceedings of the National Academy of Sciences of the United States of America 99, 16314-16318.

Desikan R., Cheung M.K., Bright J., Henson D., Hancock J.T. \& Neill S.J. (2004) ABA, hydrogen peroxide and nitric oxide signalling in stomatal guard cells. Journal of Experimental Botany 55, 205-212.

Desikan R., Last K., Harret-Williams R., Tagliavia C., Harter K., Hooley R., Hancock J.T. \& Neill S.J. (2006) Ethylene-induced stomatal closure in Arabidopsis occurs via AtrbohF-mediated hydrogen peroxide synthesis. The Plant Journal 47, 907-916.

Fan L.-M., Zhao Z. \& Assmann S.M. (2004) Guard cells: a dynamic signalling model. Current Opinion in Plant Biology 7, 537-546.

Garcia-Mata C. \& Lamattina L. (2001) Nitric oxide induces stomatal closure and enhances the plant adaptive plant responses against drought stress. Plant Physiology 126, 1196-1204.

Garcia-Mata C. \& Lamattina L. (2002) Nitric oxide and abscisic acid cross-talk in guard cells. Plant Physiology 128, 790-792.

Garcia-Mata C. \& Lamattina L. (2003) Abscisic acid, nitric oxide and stomatal closure - is nitrate reductase one of the missing links? Trends in Plant Science 8, 20-26.

Garcia-Mata C. \& Lamattina L. (2007) Abscisic acid (ABA) inhibits light-induced stomatal opening through calcium- and nitric oxide-mediated signaling pathways. Nitric Oxide 17, 143-151.

Garcia-Mata C., Gay R., Sokolovski S., Hills A., Lamattina L. \& Blatt M.R. (2003) Nitric oxide regulates $\mathrm{K}^{+}$and $\mathrm{Cl}^{-}$channels in guard cells through a subset of abscisic acid-evoked signaling pathways. Proceedings of the National Academy of Sciences of the United States of America 100, 1116-1121.

Grabov A. \& Blatt M.R. (1997) Parallel control of the inwardrectifier $\mathrm{K}^{+}$channel by cytosolic free $\mathrm{Ca}^{2+}$ and $\mathrm{pH}$ in Vicia guard cells. Planta 201, 84-95.

Grabov A. \& Blatt M.R. (1999) A steep dependence of inwardrectifying potassium channels on cytosolic free calcium concentration increase evoked by hyperpolarization in guard cells. Plant Physiology 119, 277-288.

Hetherington A.M. \& Woodward F.I. (2003) The role of stomata in sensing and driving environmental change. Nature 424, 901-908.

Israelsson M., Siegel R.S., Young J., Hashimoto M., Iba K. \& Schroeder J.I. (2006) Guard cell ABA and $\mathrm{CO}_{2}$ signaling network updates and $\mathrm{Ca}^{2+}$ sensor priming hypothesis. Current Opinion in Plant Biology 9, 654-663.

Kolla V.A. \& Raghavendra A.S. (2007) Nitric oxide is a signaling intermediate during bicarbonate-induced stomatal closure in Pisum sativum. Physiologia Plantarum 130, 91-98.

Lamotte O., Courtois C., Dobrowolska G., Besson A., Pugin A. \& Wendehenne D. (2006) Mechanisms of nitric oxide induced increase of free cytosolic $\mathrm{Ca}^{2+}$ concentration in Nicotiana plumbaginifolia cells. Free Radical Biology and Medicine 40, 13691376.

Nambara E. \& Marion-Poll A. (2005) Abscisic acid biosynthesis and catabolism. Annual Review of Plant Biology 56, 165-185.

Neill S.J., Desikan R., Clarke A. \& Hancock J.T. (2002) Nitric oxide is a novel component of abscisic acid signalling in stomatal guard cells. Plant Physiology 128, 13-16.

Neill S.J., Desikan R. \& Hancock J.T. (2003) Nitric oxide signalling in plants. New Phytologist 159, 11-35.
Neill S., Barros R., Bright J., Desikan R., Hancock J., Harrison J., Morris P., Ribeiro D. \& Wilson I. (2008) Nitric oxide, stomatal closure, and abiotic stress. Journal of Experimental Botany 59, $165-176$.

Outlaw W.H. (2003) Integration of cellular and physiological functions of guard cells. Critical Reviews in Plant Sciences 22, 503529.

Rockel P., Strube F., Rockel A., Wildt J. \& Kaiser W.M. (2002) Regulation of nitric oxide (NO) production by plant nitrate reductase in vivo and in vitro. Journal of Experimental Botany 53, 103-110.

Romano L.A., Jacob T., Gilroy S. \& Assmann S.M. (2000) Increases in cytosolic $\mathrm{Ca}^{2+}$ are not required for abscisic acid-inhibition of inward $\mathrm{K}^{+}$currents in guard cells of Vicia faba L. Planta 211, 209-217.

Saez A., Robert N., Maktabi M.H., Schroeder J.I., Serrano R. \& Rodriguez P.L. (2006) Enhancement of abscisic acid sensitivity and reduction of water consumption in Arabidopsis by combined inactivation of the protein phosphatases type 2C ABI1 and HAB1. Plant Physiology 141, 1389-1399.

She X.P., Song X.G. \& He J.M. (2004) Role and relationship of nitric oxide and hydrogen peroxide in light/dark-regulated stomatal movement in Vicia faba. Acta Botanica Sinica 46, 1292-1300.

Shimazaki K., Doi M., Assmann S.M. \& Kinoshita T. (2007) Light regulation of stomatal movement. Annual Review of Plant Biology 58, 219-247.

Sokolovski S. \& Blatt M.R. (2004) Nitric oxide block of outwardrectifying $\mathrm{K}^{+}$channels indicates direct control by protein nitrosylation in guard cells. Plant Physiology 136, 4275-4284.

Sokolovski S., Hills A., Gay R., Garcia-Mata C., Lamattina L. \& Blatt M.R. (2005) Protein phosphorylation is a prerequisite for intracellular $\mathrm{Ca}^{2+}$ release and ion channel control by nitric oxide and abscisic acid in guard cells. The Plant Journal 43, $520-529$.

Tian X. \& Lei Y. (2006) Nitric oxide treatment alleviates drought stress in wheat seedlings. Biologia Plantarum 50, 775-778.

Verslues P.E., Agarwal M., Katiyar-Agarwal S., Zhu J. \& Zhu J.K. (2006) Methods and concepts in quantifying resistance to drought, salt and freezing abiotic stress that affect plant water status. The Plant Journal 45, 523-539.

Wilkinson J.Q. \& Crawford N.M. (1991) Identification of the Arabidopsis CHL3 gene as the nitrate reductase structural gene NIA2. The Plant Cell 3, 461-471.

Wilkinson J.Q. \& Crawford N.M. (1993) Identification and characterization of a chlorate resistant mutant of Arabidopsis with mutations in both NIA1 and NIA2 nitrate reductase structural genes. Molecular Genetics and Genomics 239, 289-297.

Wilson I.D., Neill S.J. \& Hancock J.T. (2008) Nitric oxide synthesis and signalling in plants. Plant, Cell \& Environment 31, 622631.

Yan J., Tsuichihara N., Etoh T. \& Iwai S. (2007) Reactive oxygen species and nitric oxide are involved in ABA inhibition of stomatal opening. Plant, Cell \& Environment 30, 1320-1325.

Zhang A., Jiang M., Zhang J., Ding H., Xu S., Hu X. \& Tan M. (2007a) Nitric oxide induced by hydrogen peroxide mediates abscisic acid-induced activation of the mitogen-activated protein kinase cascade involved in antioxidant defense in maize leaves. New Phytologist 175, 36-50.

Zhang X., Takemiya A., Kinoshita T. \& Shimazaki K. (2007b) Nitric oxide inhibits blue light-specific stomatal opening via abscisic acid signaling pathways in Vicia guard cells. Plant Cell Physiology 48, 715-723.

Received 1 October 2008; accepted for publication 1 October 2008 